InnOvaciOnes de NegOciOs 18(35):

(c) 2021 UANL, Impreso en México (ISSN: 2007-1191)

Recepción: 9 de octubre 2020 Aceptación: 7 de enero 2021

\title{
Factores financieros de la eco innovación en procesos y organización que impactan en la productividad del sector agrícola del Valle de San Quintín, Baja California, México (Financial factors of eco-innovation in processes and organization that impact the productivity of the agricultural sector of the Valle of San Quintín Valle, Baja California, México)
}

\author{
Imelda Cuevas Merecías ${ }^{1}$, Luis Alberto Villarreal Villarreal ${ }^{2}$, María \\ Margarita Carrera Sánchez ${ }^{3}$ \& Abel Partida Puente 4 \\ Universidad Autónoma de Nuevo León \\ cuevas.imelda@uabc.edu.mx 11, luis.villarrealv@uanl.mx 2, \\ magaly_carrera@hotmail.com ${ }^{3}$, abelpartida@hotmail.com ${ }^{4}$
}

\begin{abstract}
The purpose of this preliminary study is to analyze the impact of ecoinnovation factors (processes and organization) that impact productivity in the agricultural sector of the San Quintín Valley, Baja California, Mexico. In this sense, the importance of these factors is defined, which contributes significantly to the productivity of the agricultural sector of these companies. Therefore, the central research question is defined. What are the financial factors of eco-innovation that impact the productivity of the agricultural sector of the San Quintín Valley, Baja California, Mexico? The data obtained was analyzed through statistical treatment using SPSS V-21 with a descriptive, correlational, cross-sectional and explanatory approach. The results obtained showed that only one variable is significant: eco-innovation in the organization has a positive relationship in the productivity of the agricultural sector of the San Quintín Valley, Baja California, Mexico.
\end{abstract}

Keywords: Financial factors, agricultural companies, innovation, productivity and the San Quintín Valley

JEL: 013, 031, Q1 Y Q10.

Factores financieros de la eco innovación 
Resumen: El presente trabajo de estudio preliminar tiene como objetivo analizar el impacto de los factores de la eco innovación (procesos y la organización) que impactan en la productividad del sector agrícola del Valle de San Quintín, Baja California, México. En tal sentido se define la importancia que tienen estos factores, que contribuye significativamente en la productividad del sector agrícola de estas empresas. Por lo que se define la pregunta central de investigación ¿Cuáles son los factores financieros de la eco innovación que impactan en la productividad del sector agrícola del valle de San Quintín, Baja California, México? los datos obtenidos se analizaron a través un tratamiento estadístico por medio de SPSS V-21 con un enfoque descriptivo, correlacional, transversal y explicativo. Los resultados obtenidos arrojaron que solo una variable es significativa: la eco innovación en la organización tiene relación positiva en la productividad del sector agrícola del valle de San Quintín, Baja california, México.

Palabras Clave: Factores financieros, empresas agrícolas, innovación, productividad y Valle de San Quintín.

\section{Introducción}

Bajo este contexto, el propósito de la presente investigación es probar si se cumple la hipótesis planteada: los factores financieros en los procesos y la organización con eco innovación impactan positivamente en la productividad del sector agrícola del valle de San Quintín, Baja California, México. De ahí el interés de conocer ¿Cuáles son los factores financieros de la eco innovación que impactan en la productividad del sector agrícola del valle de San Quintín, Baja California, México?

Por lo anterior, se efectuó un estudio descriptivo, correlacional, transversal y explicativo, en el cual se analizaron los factores financieros que impactan en la productividad del sector agrícola del valle de San Quintín, Baja California, México.

El trabajo está estructurado de la siguiente manera, en primer término, el marco teórico definiendo el concepto de eco innovación y cada uno de los factores financieros de la eco innovación que son en procesos y organización, la segunda fase de la investigación se indica la metodología seguida para la

\section{Factores financieros de la eco innovación}


aplicación del instrumento cuantitativo que corresponde a la encuesta, como tercera fase, se presenta en análisis de resultados estadísticos como las correlaciones, significancias, ANOVA, medias, alfas y finalmente en la cuarta fase se presentan las conclusiones dando respuesta a nuestra hipótesis, pregunta de investigación y objetivos previamente establecidos.

\section{Marco teórico}

En México la producción agrícola se ha ido desarrollando bajo un escenario de apertura dentro de su economía en los mercados nacionales y extranjeros si bien esto se ha logrado por acuerdos y tratados internacionales que se han firmado, lo que ha influido fuertemente en su desarrollo económico, tecnológico y social (Estadísticas Agrícolas de las Unidades de Riego Año agrícola 2016 - 2017, 2018).

Baja California es el estado número dos de la república mexicana, está integrado 6 municipios que son Mexicali, Tijuana, Ensenada, Rosarito, Tecate y San Quintín, las regiones agrícolas son el Valle de San Quintín y el valle de Mexicali, se tiene una superficie de 87,025.87 hectáreas sembradas de las cuales son cosechadas $83,351.16$ hectáreas, con un rendimiento de 15.33 toneladas/hectárea logrando una producción total de 1,277,655.54 toneladas que genera una valor total de la producción monetizada en 13,479,285.74 millones de pesos ( Estadísticas Agrícolas de las Unidades de Riego Año agrícola 2016 - 2017, 2018).

Los principales cultivos de la región del valle de San Quintín son Berries (Fresas, Frambuesas, Arándanos y Zarzamoras) y el tomate, no sin restarle importancia a la producción de brócoli, elotes, chiles, pimientos, calabazas, pepinos. Las empresas agrícolas a nivel mundial son quienes proveen alimentos y se encargan de satisfacer la demanda de sus consumidores, existe un deterioro ambiental y se relaciona principalmente con la sobre explotación de los recursos naturales y destrucción del medio ambiente lo que trae consigo enfermedades de los consumidores.

Cuevas, l.; Villarreal, L.; Carrera, M. \& Partida, P. 
Antecedentes de la eco innovación

La eco innovación trata sobre los cambios en los patrones de consumo y producción que se están adoptando, buscando principalmente la sostenibilidad, esta inquietud surge en los países miembros de la Organización para la Cooperación y Desarrollo Económicos (OCDE) y en países Europeos siendo ellos los que se caracterizan principalmente por poner en práctica actividades con fines sustentables, además se logra fortalecer tres pilares muy importante dentro de la economía de los países, el primero es satisfacer la demanda alimentaria a nivel internacional, la cantidad de insumos que se utilizan y principalmente la reducción de la contaminación ambiental. (Comisión Europea, 2011).

Los cambios ambientales son manifestaciones que constantemente se están dando en nuestro planeta, de acuerdo al estudio realizado por la Comisión Económica para América Latina y el Caribe (CEPAL) analizando la situación ambiental de América Latina y el Caribe consideran que es critica la situación de la administración de los recursos naturales, la contaminación del agua, aire, suelos, así como la falta de políticas gubernamentales para la regulación de estas manifestaciones, los escenarios ecológicos que se muestran actualmente tienen un gran desequilibrio.

Organismos internacionales como la CEPAL, la Organización para la Cooperación y Desarrollo Económicos (OCDE) han mantenido la lucha por preservar el equilibrio ecológico por lo que se han sumado al llamado pacto verde mundial, su principal propósito es hacer que las empresas y consumidores se comprometan a producir y consumir productos y servicios que sean verdes, es decir que generen un menor impacto ambiental (UNESCAP, 2008).

Eco innovación es un término que ha ido evolucionando a través del tiempo, en 1989 se dio a conocer por primera vez el termino economía verde, este concepto consistió en una evolución que no solo involucra la parte ecológica, también integra la parte económica social que involucra a los consumidores y el comportamiento que manifiestan, este tipo de economía busca una sostenibilidad entre la necesidades de alimentar a la sociedad y la necesidad de mantener un equilibrio ecológico por parte de las empresas al utilizar los recursos naturales (UNIDO, 2011).

\section{Factores financieros de la eco innovación}


La economía verde, es un elemento esencial para el desarrollo sostenible de la economía de los países, debido a que no solo considera la parte económica sino que también considera el medio ambiente y busca que los procesos que se aplique dentro de la empresas de cualquier sector sean más eficientes en relación a la cantidad de recursos naturales que utilizan, si bien es importante mencionar que estas iniciativas pueden lograr que las empresas aumenten su competitividad y esto les permita generar ganancias económicas (UNIDO, 2011).

El Manual de OSLO establece factores en los cuales mide la eco innovación dentro de ciertas empresas que se encuentran en América Latina y el Caribe, el cual se integra por la eco innovación en procesos, eco innovación en productos, eco innovación en la organización, en la comercialización, eco innovación social, eco innovación en el flujo de materiales, así mismo hace diferencia entre la eco innovación que él considera incremental, eco innovación disruptiva y la eco innovación radical. La comunidad europea se distingue por ser quien ha implementado los procesos productivos más sustentables.

El trabajo de investigación realizado por Abreu y Acosta (2016) definen los factores que impulsan a las empresas a tomar la decisión de aplicar la eco innovación, clasifican a estos factores en tres grupos, el primer grupo está relacionado con la oferta, el segundo con la demanda y el tercero con las políticas ambientas que se tienen en cada una de las regiones, analizando estos tres grupo se determina que las empresas que vayan a adoptar estas prácticas ecológicas tengan la capacidad para poder combinar la eco innovación con sus procesos, con sus productos y con los objetivos ambientales que cada organización tenga planteados.

En 2000, Rennings argumentaba que la eco innovación en procesos es generada principalmente por las presiones que se generan en el ambiente externo, mientras que la eco innovación en productos es impulsada por los beneficios económicos que proporciona el mercado. (Rovira, Patiño y Schaper, 2017, p. 27)

El invertir en procesos ecológicos no es sencillo y no solo por el grado de complejidad que tienen el crear esa cultura dentro de la organización, sino por la cantidad de efectivo que se tiene que desembolsar para poder estructurar todos los eslabones de la empresa y así lograr una eficiente

Cuevas, l.; Villarreal, L.; Carrera, M. \& Partida, P. 
aplicación de procesos más ecológicos, sin embargo, es importante considerar que esta práctica requiere de lata inversión bastante alta. (Abreu y Acosta, 2016, p.5).

\section{Eco innovación en la organización}

Este proceso cosiste en involucrar a todos los integrantes de la organización para que adopten prácticas ecológicas, estas prácticas van desde la creación de políticas, implementación de procesos productivos más ecológicos, creando una cultura de eco innovación dentro de la organización. Considerando la aparte social que incluye a los consumidores y sus cambios de hábitos en la demanda de productos alimenticios más limpios, ya que son ellos quienes generan esa presión social para que las empresas de alimentos ofrezcan productos más sanos y al mismo tiempo más sustentables.

Las practicas ecológicas que son puestas en marcha según el manual de Oslo es la creación de planes para prevenir la contaminación, planes para reducir la cantidad de contaminantes, el cumplimiento de normar ecológicas establecida en cada uno de los países, la gestión de la cadena de suministro, que no solo se refiere a la utilización de insumos ecológicos sino también a la disponibilidad de los proveedores para poder ofrecer insumos más ecológicos.

\section{Teoría evolucionista}

Esta teoría es del autor Neoschumpeter y fue desarrollada por Nelson y Winter (1977), toma en cuenta principalmente el comportamiento de la empresa ante cada una de las situaciones de innovación que están en su ambiente competitivo, en el cual la empresa tendrá un buen desenvolvimiento de acuerdo a la trayectoria o a la experiencia que haya adquirido, así como el reconocimiento que haya creado por medio de su ventaja competitiva, esta teoría hace mención con respecto a la percepción que tiene el cliente y a las estrategias con las que ha trabajado la empresa, tomando en cuenta cuatro áreas principales que son el área de producción, distribución, producto y la comercialización que se le desee dar al producto.

En 2012, Pereira y Vence enfatizan que la teoría evolucionista consiste en una fase de transición dentro de la innovación tecnológica y esto genera

\section{Factores financieros de la eco innovación}


cierto grado de incertidumbre considerando los beneficios que se obtendrán dentro de la organización. (Abreu y Acosta, 2016, p.8)

Eco innovación en México

De acuerdo con el trabajo de estudio realizado por García et al, 2012, define que dentro de los principales problemas del sector agrícola se ha detectado que las enfermedades, el abastecimiento de alimentos, el tratamiento de aguas y el uso de fertilizantes son los principales problemas que afectan a este sector en México y los productores interesados preocupados en no generar mayores pérdidas en la producción.

En México el $85 \%$ de los suelos destinados a la agricultura se encuentran degradados, por una parte lo que contribuye a la contaminación ambiental son los fertilizantes utilizados para maximizar la producción agrícola y por otro lado la demanda de alimentos que ha hecho que se incorporen los alimentos transgénicos para garantizar una mayor producción, pero al mismo tiempo hace que estas empresas pongan en riesgo la ecología del país por el gran impacto que esto representa para el medio ambiente y que el daño sería irreversible para México (Greenpeace México, 2009).

El mundo busca una intensificación sostenible que permita un equilibrio entre la producción y la demanda alimentaria, por lo tanto, se trata de crear una negociación social que permita una innovación institucional y el manejo adaptativo de los recursos naturales. Por lo tanto los propietarios de granjas así como los profesionales que están involucrados deben demostrar la capacidad de hacer frente al cambio que se está presentando, así como garantizar las ganancias para sus grupos de interés basando cada uno de sus procesos en actividades con valores, responsabilidad e inteligencia (Struik y Kuyper 2017).

China es un país que se caracteriza por alta densidad de población, numerosas de empresas industriales lo que genera una gran cantidad de emisiones de contaminantes no obstante a esto este país de está preocupando por cambiar esa imagen internacional que se tiene, del mismo modo les permite obtener subsidios, impuestos ambientales, subsidio a la producción y un comercio especial, lo que les permite mejorar sus ventajas competitivas, este trabajo no solo se está logrando por la iniciativa de los dueños de las empresas sino que también por el apoyo que el gobierno les provee a las

Cuevas, I.; Villarreal, L.; Carrera, M. \& Partida, P. 
empresas que se están involucrando, los proveedores que también forman parte de esta cadena evolutiva que se preocupa por ofrecer productos verdes (Peng, Yu y Wei, 2018).

\section{Estrategia nacional de producción y consumo sustentable en México}

En el año de 2012 durante el gobierno de Felipe Calderón, se buscó el desarrollo humano y sustentable, considerando temas de gran relevancia, como lo es la utilización de enérgicas renovables, prevención y gestión de los residuos, control de emisiones de gases efecto invernadero (administrados de manera voluntaria por cada una de las empresas), liderazgo ambiental, dentro de estos lineamientos se ha tenido muy buena respuesta y en gran medida es gracias a la conciencia por la cantidad de desastres naturales que se han presentado en México y otros países (Plan nacional de desarrollo, 2007).

Este modelo busca una sustentabilidad en la utilización de los recursos naturales buscando de esta manera no comprometer los recursos naturales de las generaciones futuras, la adopción de procesos productivos sustentables permite que las empresas involucradas fomentan el crecimiento sostenible de la economía, generan empleos, crean patrones de innovación generando un menor impacto ambiental beneficiando a la sociedad.

La producción y consumo nacional en México tiene una particular característica y es que los hábitos de consumo se hacen sin alguna consideración, no se considera el origen de los productos, la durabilidad que tendrá, sin importar los procesos de producción, la calidad de los insumos o la sustentabilidad que representa dentro del proceso de comercialización. La oferta, demanda y procesos de producción que no son ecológicos ha ocasionado un uso desmedido de los recursos naturales buscando solo satisfacer las necesidades del mercado demandante.

La producción y consumo sustentable busca implementar políticas ambientales que incentiven a los sectores económicos de México a la adopción de procesos productivos sustentables para que haya un equilibrio entre la satisfacción de las necesidades de consumo y el uso de los recursos naturales.

México es un país que cuenta con una diversidad de recursos naturales, pero también es un país que no hace uso racional de los recursos naturales al producir un servicio o un producto, el $78 \%$ de la contaminación es generada

\section{Factores financieros de la eco innovación}


por diversas actividades propias de los mexicanos, mientras que el $7 \%$ de ellas es generado por las actividades de producción de alimentos y servicios. De 2007 a 2009 se incrementó un 11\% la demanda y el uso del recurso hídrico donde el $77 \%$ se destinó al uso agrícola y el $23 \%$ fue destinado al resto de otras actividades comerciales.

Crute y Muir (2011). El incremento de la productividad de alimentos actualmente está relacionado con el uso de las innovaciones tecnológicas y al mismo tiempo busca el equilibrio sostenible y el uso eficiente de cada uno de los recursos naturales (agua, suelo, aire, etc.). La productividad se ve afectada por la calidad del agua, condiciones climáticas como las temperaturas, plagas, enfermedades, la utilización de agroquímicos por lo que se requiere una mayor inversión para incrementar la productividad. Dentro de esta investigación se analiza el impacto que puede generar la tecnología e investigación en el desarrollo agrícola.

Crute y Muir 2011, la demanda de alimentos que se tiene actualmente, el sector agrícola mundial tendrá que incrementar su productividad en la producción de alimentos actuando siempre de manera sostenible considerando los recursos que utilizara, el desarrollo tecnológico de los cultivos permitirá un mayor rendimiento en los cultivos y finalmente esto permitirá el uso eficiente de los recursos tales como el agua y el suelo.

Dziallasa y Blind (2019). Las innovaciones se miden de acuerdo a la eficiencia de la utilización de los recursos y el desempeño que tienen las empresas, al tener una cultura de innovación permite mejorar el desarrollo de la organización.

Jürgen y González (2020). Considerando la demanda alimentaria y la disponibilidad de recursos para el sector agrícola en América Latina se debe mencionar que existe una fuerte dependencia del uso de los recursos y el precio de los productos, el agua es uno de los recursos más limitado en estas regiones lo que trae consigo deficiencias al momento de producir, golpeado fuertemente la economía de estas regiones.

Planteamiento del problema

En lo que respecta al sector agrícola se destaca el sector agrícola en las diferentes regiones en México, ya que generan empleo y una gran derrama

Cuevas, I.; Villarreal, L.; Carrera, M. \& Partida, P. 
económica en el uso de insumos (Villarreal et al. 2005). Por tal razón se propone esta investigación debido al crecimiento poblacional que tiene México, la demanda de alimentos, las políticas gubernamentales que se crean para el cuidado y el uso sustentable de los recursos naturales, ya que diferentes estudios señalan que debe existir un equilibrio entre estos.

Objetivo

Determinar los Factores financieros de la eco innovación que impactan en la productividad del sector agrícola del valle de San Quintín, B.C., Baja California, México?

\section{Metodología}

\section{Población y Tamaño de la Muestra}

La investigación de campo consistió en aplicar una encuesta a los productores agrícolas de acuerdo al directorio de la Secretaria de Agricultura y Desarrollo Rural utilizada correspondiente al año 2017.

Para establecer el tamaño de la muestra se utilizó la fórmula para el cálculo de una muestra aleatoria simple, con un coeficiente de confianza del $5 \%$ y un error estadístico máximo deseado del $10 \%$, por ser el más usado en las ciencias sociales. Utilizando los datos de la "n" optima la cantidad mínima será de 30 encuestas a realizar para la población de estudio $\mathrm{N}$ total $=66$ encuestas a realizar, "p" será igual a "q" en la muestra y será de $50 \%$ para que la probabilidad de éxito o fracaso no tenga sesgo. Con un $a=95 \%$, la Z por tablas es de 1.96 logrando obtener un total de 35 encuestas para la presente investigación.

Determinación de la muestra aleatoria de acuerdo a la siguiente fórmula:

$$
\text { n optima }=\frac{\left(N \text { total }{ }^{*} p^{*} q\right)}{\frac{(N \text { total }-1) E^{2}+p^{*} q}{Z^{2}}}
$$

\section{Factores financieros de la eco innovación}




\section{Resultados}

Para obtener la respuesta a esta investigación se analizó la base de datos a través un tratamiento estadístico por medio de SPSS V-21 con un enfoque descriptivo, correlacional, transversal y explicativo. En el modelo se han definido las siguientes variables independientes que son: (X1), Procesos y $(X 2)$, organización y como variable dependiente la productividad $(Y)$

Tabla 1. Variables del modelo

\begin{tabular}{lc}
\hline Variables & Descripción \\
\hline $\mathrm{X} 1$ & Procesos \\
$\mathrm{X} 2$ & Organización \\
$\mathrm{Y}$ & Productividad \\
\hline
\end{tabular}

Fuente: Elaboración propia.

Operacionalización de las Variables

$$
\hat{\mathbf{Y}} 1=\beta 0+\beta 1 X 1+\beta 2 X 2+e
$$

Cabe señalar que se obtuvo un Alfa de Cronbach de .960 basado en 51 ítems considerandos en el instrumento de medición en función de las variables independientes y dependiente incluida en el modelo general, para lo cual se utilizó el paquete estadístico SPSS V-21.

Tabla 2. Alfa de Cronbach de todas las variables general del instrumento de investigación

\begin{tabular}{cc}
\hline Alfa de Cronbach & N de elementos \\
\hline .960 & 33 \\
\hline
\end{tabular}

Fuente: Elaboración propia.

Cabe señalar que se obtuvieron los alfas de cada una de las variables tanto independientes como la variable dependiente, en la variable $X 1$ se obtuvo un alfa de .908, mientras que en la variable X2 fue de .936 y finalmente el alfa de la variable dependiente fue de .953 de acuerdo como se muestra en la tabla 3.

Cuevas, l.; Villarreal, L.; Carrera, M. \& Partida, P. 
Tabla 3. Análisis del alfa de Cronbach por cada una de las variables

\begin{tabular}{cllccc}
\hline Variables & \multicolumn{2}{c}{ Descripción } & & $\begin{array}{c}\text { Alfa de } \\
\text { Cronbach }\end{array}$ & $\begin{array}{c}\mathrm{N} \text { de } \\
\text { elementos }\end{array}$ \\
\hline $\mathrm{X} 1$ & $\begin{array}{l}\text { Factores financieros de la eco } \\
\text { innovación en procesos }\end{array}$ & .908 & 11 \\
$\mathrm{X} 2$ & $\begin{array}{l}\text { Factores financieros de la eco } \\
\text { innovación en la organización }\end{array}$ & .936 & 11 \\
$\mathrm{Y}$ & $\begin{array}{l}\text { Productividad del Sector Agrícola del } \\
\text { valle de San Quintín, Baja california, } \\
\text { México. }\end{array}$ & .953 & 11 \\
\hline
\end{tabular}

Fuente: Elaboración propia.

Análisis estadístico

Regresión lineal múltiple

Se definieron las variables independientes (X1 y X2) con una muestra de 35 encuestas en relación con la variable dependiente Productividad del sector agrícola del Valle de San Quintín, esto con la finalidad de obtener un análisis la regresión lineal múltiple, cada uno de los valores fueron analizados dentro del programa de SPSS-V21 logrando obtener cantidades precisas para cada una de las variables.

Una vez obtenida la base de datos se procesaron dentro del programa estadístico SPSS-v21 para realizar el análisis estadístico de cada una de las variables tanto independientes como la variable dependiente, la correlación se considera alta cuando tiene un rango $(>0.50)$ entre la variable es indicativo de colinealidad lo cual se puede observar en la tabla 4, lo cual confirma la relación entre la variable independiente ( $Y=$ Productividad del sector agrícola) y la variable independiente $(\mathrm{X} 2)$ es significativa a través del rango de todos los valores considerando que nuestro modelo es una regresión lineal múltiple logrando una contrastación de la hipótesis general es distinto a cero por lo tanto se acepta que hay un nivel de correlacional alto, fuerte y positivo.

En 1988, Cashin afirma que los valores de los coeficientes de correlación obtenidos son de práctica utilidad dentro de la variable X2 que corresponde a los factores financieros de la eco innovación en la organización

\section{Factores financieros de la eco innovación}


se tiene una muy alta practica debido a que se encuentran por encima de .50 y .70, a diferencia de los que se encuentran entre .20 y .49 (Carrera, 2016).

Tabla 4. Inter-ítem correlaciones Matriz Y1

\begin{tabular}{|c|c|c|c|c|}
\hline & & $\begin{array}{l}\text { Factores } \\
\text { financieros de } \\
\text { la eco } \\
\text { innovación en } \\
\text { procesos. }\end{array}$ & $\begin{array}{l}\text { Factores } \\
\text { financieros de } \\
\text { la eco } \\
\text { innovación en } \\
\text { la } \\
\text { organización. }\end{array}$ & $\begin{array}{l}\text { Productividad } \\
\text { del sector } \\
\text { agrícola del } \\
\text { valle de San } \\
\text { Quintín, BC, } \\
\text { México. }\end{array}$ \\
\hline $\begin{array}{l}\text { correlación } \\
\text { de Pearson }\end{array}$ & $\begin{array}{l}\text { Factores financieros de } \\
\text { la eco innovación en } \\
\text { procesos } \\
\text { Factores financieros de } \\
\text { la eco innovación en la } \\
\text { organización }\end{array}$ & $\begin{array}{c}1 \\
.651^{* *}\end{array}$ & $.651^{* *}$ & $.776^{* *}$ \\
\hline & $\begin{array}{l}\text { Productividad del sector } \\
\text { agrícola del valle de San }\end{array}$ & $.517^{\star *}$ & $.776^{* *}$ & \\
\hline Siq. & $\begin{array}{l}\text { Quintín, BC, México } \\
\text { Factores financieros de }\end{array}$ & & & 1 \\
\hline (unilateral) & $\begin{array}{l}\text { la eco innovación en } \\
\text { procesos } \\
\text { Factores financieros de } \\
\text { la eco innovación en la } \\
\text { organización }\end{array}$ & .000 & .000 & .001 \\
\hline \multirow{4}{*}{$\mathrm{N}$} & $\begin{array}{l}\text { Productividad del sector } \\
\text { agrícola del valle de San } \\
\text { Quintín, BC, México }\end{array}$ & .001 & .000 & \\
\hline & $\begin{array}{l}\text { Factores financieros de } \\
\text { la eco innovación en } \\
\text { procesos }\end{array}$ & 35 & 35 & 35 \\
\hline & $\begin{array}{l}\text { Factores financieros de } \\
\text { la eco innovación en la } \\
\text { organización }\end{array}$ & 35 & 35 & 35 \\
\hline & $\begin{array}{l}\text { Productividad del sector } \\
\text { agrícola del valle de San } \\
\text { Quintín, BC, México }\end{array}$ & 35 & 35 & 35 \\
\hline
\end{tabular}

Fuente: Elaboración propia.

Cuevas, I.; Villarreal, L.; Carrera, M. \& Partida, P. 
Así mismo se realizó la prueba de normalidad de los datos, la cual se realizó mediante la prueba de Kolmogorov-Smirnov considerando que se tienen 35 encuestas preliminares para el análisis estadístico, la prueba de normalidad de los datos, los cuales se realizaron mediante la prueba Kolmogorov-Smirnov, dentro de esta prueba podemos conocer la bondad de ajuste de la distribución normal de los datos y se puede observar en la tabla número 5.

Tabla 5. Prueba de kolmogorov-Smirnov para una muestra

\begin{tabular}{|c|c|c|c|c|}
\hline \multicolumn{5}{|c|}{ Prueba de Kolmogorov-Smirnov para una muestra } \\
\hline & & $\begin{array}{l}\text { Productividad del } \\
\text { sector agrícola del } \\
\text { Valle de San } \\
\text { Quintín, Baja } \\
\text { California, México. }\end{array}$ & $\begin{array}{l}\text { Factores } \\
\text { financieros de la } \\
\text { eco innovación } \\
\text { en procesos }\end{array}$ & $\begin{array}{l}\text { Factores } \\
\text { financieros de la } \\
\text { eco innovación } \\
\text { en la } \\
\text { organización }\end{array}$ \\
\hline \multicolumn{2}{|l|}{ N } & 35 & 35 & 35 \\
\hline \multirow{2}{*}{$\begin{array}{l}\text { Parámetros } \\
\text { normalesa,b }\end{array}$} & Media & 4.4883 & 3.6935 & 4.1688 \\
\hline & $\begin{array}{l}\text { Desviación } \\
\text { típica }\end{array}$ & 0.61027 & 0.83526 & 0.71076 \\
\hline \multirow{3}{*}{$\begin{array}{l}\text { Diferencias } \\
\text { más extremas }\end{array}$} & Absoluta & .201 & .173 & .134 \\
\hline & Positiva & .201 & .079 & .121 \\
\hline & Negativa & -.195 & -.173 & -.134 \\
\hline \multicolumn{2}{|c|}{ Z de Kolmogorov-Smirnov } & 1.188 & 1.021 & .792 \\
\hline \multicolumn{2}{|c|}{ Sig. asintót. (bilateral) } & .119 & .249 & .558 \\
\hline
\end{tabular}

a. La distribución de contraste es la Normal.

b. Se han calculado a partir de los datos.

Fuente: Elaboración propia.

De acuerdo con los resultados se presentan las medias y las desviaciones estándar en la tabla 6 , los resultados obtenidos en la regresión lineal de las variables factores financieros de la eco innovación en procesos y organización son los que se describen a continuación. 
Tabla 6. Medias y desviación estándar

\begin{tabular}{|c|c|c|c|}
\hline & Media & Desviación estándar & $\mathrm{N}$ \\
\hline $\begin{array}{l}\text { Productividad del } \\
\text { sector agrícola del } \\
\text { Valle de San } \\
\text { Quintín, Baja } \\
\text { California, México. }\end{array}$ & 4.4883 & 0.61027 & 35 \\
\hline $\begin{array}{l}\text { Factores financieros } \\
\text { de la eco innovación } \\
\text { en procesos }\end{array}$ & 3.6935 & 0.83526 & 35 \\
\hline $\begin{array}{l}\text { Factores financieros } \\
\text { de la eco innovación } \\
\text { en la organización }\end{array}$ & 4.1688 & 0.71076 & 35 \\
\hline
\end{tabular}

Fuente: Elaboración propia.

El valor que se observa en la Durbin-Watson, comprueba la independencia de residuales y muestra la presencia de residuales y presencia de auto correlación de primer orden en los residuales en una regresión, en el presente estudio el valor Durbin Watson se define que es 1.886 por lo tanto se define que no hay auto correlación en el modelo de estudio, considerando una regresión lineal dentro del valor aceptado del rango establecido de 1.5 a 2, además se obtienen un coeficiente de correlación aceptada de ( $r$ ) que corresponde a $77.6 \%$ para el modelo de la presente investigación, los coeficientes de determinación de $\left(r^{2}\right) 60.2 \%$, con una $\left(r^{2}\right)$ ajustado de $57.7 \%$ se puede apreciar en la siguiente tabla 7 .

Cuevas, I.; Villarreal, L.; Carrera, M. \& Partida, P. 
Tabla 7. Resultados de la regresión lineal

\begin{tabular}{|c|c|c|c|c|c|c|c|c|c|c|}
\hline \multicolumn{11}{|c|}{ Resumen del modelob } \\
\hline \multirow{6}{*}{$\begin{array}{c}\text { Model } \\
0 \\
1\end{array}$} & \multirow{5}{*}{$\mathrm{R}$} & \multirow{5}{*}{$\begin{array}{c}\mathrm{R} \\
\text { cuadrad } \\
0\end{array}$} & \multirow{5}{*}{$\begin{array}{c}R \\
\text { cuadrad } \\
0 \\
\text { corregid } \\
a\end{array}$} & \multirow{5}{*}{$\begin{array}{c}\text { Error típ. } \\
\text { de la } \\
\text { estimació } \\
n\end{array}$} & \multicolumn{5}{|c|}{ Estadísticos de cambio } & \multirow{4}{*}{$\begin{array}{c}\text { Durbir } \\
- \\
\text { Watso }\end{array}$} \\
\hline & & & & & Cambio & & & & & \\
\hline & & & & & & & & & $\mathrm{Sig}$ & \\
\hline & & & & & cuadrad & Cambi & $\mathrm{gl}$ & gl & Cambi & \\
\hline & & & & & 0 & 0 en $F$ & 1 & 2 & 0 en $\mathrm{F}$ & $\mathrm{n}$ \\
\hline & $\begin{array}{c}.776 \\
\mathrm{a}\end{array}$ & .602 & .577 & 4.36721 & .602 & 24.167 & 2 & 32 & .000 & 1.886 \\
\hline
\end{tabular}

a. Variables predictores: (Constante), Factores financieros de la eco innovación en procesos, Factores financieros de la eco innovación en la organización.

b. Variable dependiente: VD1 Productividad del sector agrícola del valle de San Quintín, Baja California, México.

Fuente: Elaboración propia.

En la tabla 8 , se define el modelo y la significancia entre las variables además se puede observar que no muestra una multicolinealidad entre las variables independientes mostrando una colinealidad aceptable con un marcado índice del factor de inflación de varianza (VIF), debido que el valor debe ser inferior de 10.

Tabla 8. Índice de factor de la influencia de la varianza para $Y$

\begin{tabular}{|c|c|c|c|c|c|c|c|c|c|c|}
\hline \multicolumn{11}{|c|}{ Coeficientes $^{\mathrm{a}}$} \\
\hline \multirow{2}{*}{ Modelo } & \multicolumn{2}{|c|}{$\begin{array}{c}\text { Coeficientes } \\
\text { no } \\
\text { estandarizad } \\
\text { os }\end{array}$} & \multirow{2}{*}{$\begin{array}{c}\text { Coeficient } \\
\text { es } \\
\text { tipificados } \\
\text { Beta }\end{array}$} & \multirow{2}{*}{$t$} & \multirow{2}{*}{ Sig } & \multicolumn{3}{|c|}{ Correlaciones } & \multicolumn{2}{|c|}{$\begin{array}{l}\text { Estadísticos } \\
\text { de colinealidad }\end{array}$} \\
\hline & B & $\begin{array}{l}\text { Error } \\
\text { típ. }\end{array}$ & & & & $\begin{array}{c}\text { Orde } \\
\text { n } \\
\text { cero }\end{array}$ & $\begin{array}{c}\text { Parci } \\
\text { al }\end{array}$ & $\begin{array}{c}\text { Semi } \\
\text { parci } \\
\text { al }\end{array}$ & $\begin{array}{c}\text { Toleran } \\
\text { cia }\end{array}$ & FIV \\
\hline $\begin{array}{l}1 \text { (Constant } \\
\text { e) } \\
\text { Factores } \\
\text { financiero } \\
\text { s de la }\end{array}$ & $\begin{array}{c}18.7 \\
52\end{array}$ & $\begin{array}{c}4.49 \\
4\end{array}$ & & $\begin{array}{c}4.17 \\
3\end{array}$ & $\begin{array}{c}.00 \\
0\end{array}$ & & & & & \\
\hline $\begin{array}{l}\text { eco } \\
\text { innovació } \\
\text { n en } \\
\text { procesos }\end{array}$ & .015 & .107 & .021 & .143 & $\begin{array}{c}.88 \\
7\end{array}$ & .517 & .025 & .016 & .576 & $\begin{array}{c}1.73 \\
7\end{array}$ \\
\hline
\end{tabular}

Factores financieros de la eco innovación 
Factores

financiero

s de la

eco

innovació

$.654 \quad .126 \quad .762$

$\begin{array}{cc}5.18 & .00 \\ 0 & 0\end{array}$

$.776 \quad .675$

$.578 \quad .576$

1.73
7

n en la

organizaci

ón

a. Variable dependiente: Productividad del sector agrícola del Valle de San Quintín, Baja California, México.

Fuente: Elaboración propia.

El análisis de varianza ANOVA compara las medias de cada variable para poder definir que existe una dependencia entre las variables independientes, por lo que se determina que el modelo aceptado en la tabla 9 muestra la independencia contra las otras variables por tener un valor inferior del $5 \%$ de significancia.

Tabla 9. Análisis de varianza ANOVA para Y1

\begin{tabular}{lccccc}
\hline \multicolumn{5}{c}{ ANOVA $^{\mathrm{a}}$} \\
\hline Modelo & Suma de cuadrados & gl & cuadrática & $\mathrm{F}$ & Sig. \\
1 Regresión & 921.850 & 2 & 460.925 & 24.167 & $.000^{\mathrm{b}}$ \\
Residual & 610.321 & 32 & 19.073 & & \\
$\quad$ Total & 1532.171 & 34 & & & \\
\hline
\end{tabular}

a. Variable dependiente: Productividad del sector agrícola del Valle de San Quintín, Baja California, México.

b. Variables predictoras: (Constante), Factores financieros de la eco innovación en procesos y Factores financieros de la eco innovación en la organización.

Fuente: Elaboración propia.

Con base a los resultados obtenidos a las significancias de la T-Student referido en la tabla 10, se puede observar un error estimado menor al $5 \%$ se puede definir que la variable que impacta significativamente la productividad del sector agrícola es la variable X2 = Factores financieros de la eco innovación en la organización el cual arroja un valor de significancia de .00\%.

\section{Cuevas, l.; Villarreal, L.; Carrera, M. \& Partida, P.}


En el presente estudio la ecuación lineal para la variable dependiente $=$ Productividad del sector agrícola del Valle de San Quintín, Baja California, México, se define de la siguiente manera.

T-Student

$$
\hat{Y} 1=18.752+0.654+\varepsilon
$$

De las dos variables independientes que se consideraron en la presente investigación se quedó excluida una variable en el modelo para la variable dependiente $Y 1$ = Productividad del sector agrícola del Valle de San Quintín. Esta variable corresponde a X1 = Factores financieros de la eco innovación en procesos.

Considerando las significancias que se obtuvieron de la T- Student esas variables arrojaron un valor estimado superior del $5 \%$, por lo que se determina que una variable que rechazada.

\section{Discusión}

Logrando dar respuesta a la Pregunta, hipótesis y Objetivos de investigación los resultados obtenidos son los siguientes.

\section{$\underline{\text { Pregunta General de Investigación }}$}

Para el desarrollo de la presente investigación se establece la siguiente pregunta central de investigación

¿Cuáles son los factores financieros de la eco innovación que impactan en la productividad del sector agrícola del valle de San Quintín, Baja California, México?

De acuerdo a los resultados los factores financieros de la eco innovación en la organización es el factor que impacta positivamente en la productividad del sector agrícola del Valle de San Quintín.

\section{Factores financieros de la eco innovación}


$\underline{\text { Preguntas específicas de investigación }}$

¿Cuál es el impacto que generan la eco innovación en procesos en la productividad del sector agrícola del valle de San Quintín, Baja California, México?

Este factor no interviene significativamente en la productividad del sector agrícola del valle de San Quintín.

¿Cuál es el impacto que genera la eco innovación en la organización en la productividad del sector agrícola del valle de San Quintín, Baja California, México?

Los factores financieros de la eco innovación en la organización impacta significativamente con un $65.4 \%$ en la productividad del sector agrícola del valle de San Quintín, Baja California, México.

Objetivo General de Investigación

Determinar los Factores financieros de la eco innovación que impactan en la productividad del sector agrícola del valle de San Quintín, Baja California, México a fin de proponer estrategias que permitan la sustentabilidad de los recursos naturales y la satisfacción de la demanda alimenticia.

Se analizaron los factores que impactan significativamente en la productividad del Sector Agrícola del Valle de San Quintín, en el cual los factores financieros de la eco innovación en la organización impactan positivamente en la productividad en el Sector Agrícola del Valle de San Quintín.

Objetivos específicos de la investigación siguientes.

La presente investigación establece como objetivos específicos los

Cuevas, I.; Villarreal, L.; Carrera, M. \& Partida, P. 
Determinar y analizar los factores financieros de la eco innovación de los procesos que impacta en la productividad del sector agrícola del valle de San Quintín, Baja California, México

No hubo impacto en la productividad de las empresas agrícolas del Valle de San Quintín.

Determinar y analizar los factores financieros de la eco innovación en la organización que impacta en la productividad del sector agrícola del valle de San Quintín, Baja California, México.

Los factores financieros del eco innovación en la organización impactan significativamente en la productividad del sector agrícola del Valle de San Quintín, baja California, México.

Los resultados obtenidos dentro de esta investigación coindicen con Bergset (2018), quien afirma que el desarrollo de la economía verde se da gracias a las empresas que emplean y adoptan prácticas ecológicas y en especial dentro de sus procesos considerándolos ecológicos, por tal motivo es importante que las empresas tengan un financiamiento directo para esta actividad para poder lograr culminar este cambio dentro de la organización.

La eco innovación es un proceso que se ha ido incorporando dentro de la sustentabilidad, de acuerdo con las investigaciones realizadas por Hazarica y Zhang (2019), afirman que esto se da de acuerdo a las limitaciones y disponibilidad de los recursos dentro del entorno natural, lo que conlleva a que las empresas adopten este tipo de prácticas, y finalmente los resultado obtenidos concuerdan en que las organizaciones del Valle de San Quintín están adoptando estas prácticas ecológicas en relación a la disponibilidad de los recursos naturales de esta región logrando cumplir con las expectativas del desarrollo sustentable.

\section{Referencias}

Abreu, E. y Acosta, J.. (2016). Eco innovación / Eco innovation (tesis de grado de doctor). Bergset, L. (2018). Green start-up finance - where do particular ones lie?. International Journal of Entrepreneurial Behavior \& Research, 24(2). 451-575.

Cashin, W.E. (1988). Students Rattings of Teaching: A Summary of the Research. Kansas State University. Kansas: Center for Faculty Evaluation \& Devolment.

\section{Factores financieros de la eco innovación}


Comisión Europea (2012). Eurobarometer survey: SMEs are important for a smooth transition to a greener economy. (MEMO/12/218). Bruselas: Comisión Europea.

Crute, I., \& Muir, J. (2011). Improving the productivity and sustainability of terrestrial and aquatic food production systems: Future perspectives. The Journal of Agricultural Science, 149(1). 1-7.

Dziallas, M. y Blind K. (2019). Innovation indicators throughout the innovation process: An extensive literature analysis. Technovation, 89.

García-Gutiérrez, C., y Rodríguez-Meza, G. (2012). Problemática y riesgo ambiental por el uso de plaguicidas en Sinaloa. Ra Ximhai, 8(3b). 1-10.

Greenpeace (2009). La destrucción de México La realidad ambiental del país y el cambio climático. México.

Hazarika, N. y Zhang, X. (2019). Factors that drive and sustain eco-innovation in the construction industry: The case of Hong Kong. Journal of cleaner Production, 238.

Mahlknecht, J., González-Bravo, R., \& Loge, F. J. (2020). Water-Energy-Food Security: A Nexus Perspective of the Current Situation in Latin America and the Caribbean. Energy.

Nelson, R. y S. Winter (1977). In search of a useful theory of innovation. Research Policy, (6). 36-76.

Peng, B., Tu, Y. and Wei, G. (2018). Can Environmental Regulations Promote Corporate Environmental Responsibility? Evidence from the Moderated Mediating Effect Model and an Empirical Study in China. Sustainability.

Rovira, S., Patiño, J. y Scharper, M. (2017). Eco innovación y producción verde: Una revisión sobre las políticas de América Latina y el Caribe.

Secretaría de Hacienda y Crédito Público (2007). Plan nacional de desarrollo 2007-2012. Ciudad de México.

Secretaría del medio ambiente y recursos naturales (2018). Estadísticas agrícolas de las unidades de riego año 2016-2017.

Struik, P. y Kuyper, T.(2017). Sustainable intensification in agriculture: the richer shade of green. A review. Agron. Sustain. Dev. 37(39).

United Nations Economic and Social Commission for Asia and the Pacific (2008). Economic and Social Survey of Asia and the Pacific.

United Nations Industrial Development Organization (2011). From Labour to Resource Productivity. Vienna.

United Nations Industrial Development Organization (2011). Green Industry: Policies for supporting Green Industry. Vienna.

Villareal, G. y Dena, G., (2005). Importancia del Sector Hortícola en el Sur de Sonora, Implicaciones en la Asignación de Recursos e Impacto Social. Memorias XIX Congreso Internacional en Administración de Empresas Agropecuarias. Guanajuato, Gto.

\section{Cuevas, l.; Villarreal, L.; Carrera, M. \& Partida, P.}

